Gold wird in den neuesten Messungen ${ }^{3}$ mit $\sigma_{0}=$ $97,6 \pm 0,5$ barn angegeben. Diese Messungen wurden zwischen 0,001 und $0,005 \mathrm{eV}$ durchgeführt, wo der Einfluß der Resonanz vernachlässigt werden kann, und auf $E_{0}$ extrapoliert. Der obige Wert ist deshalb der reine $\mathbf{1} / v$-Teil des Querschnittes. Daraus folgt mit Hilfe von (4) $R=1231 \pm 15$ barn.

Da die Goldfolie relativ dick war (beträgt doch der Absorptionsquerschnitt im Maximum der Resonanz 20000 barn), mußte eine Korrektur für die Selbstabsorption angebracht werden. Die tatsächlich gemessene Resonanzaktivierung beträgt

$$
k \int_{0}^{\infty} \frac{\sigma_{\mathrm{a} \text { res }}(E)}{\sigma_{\mathrm{t}}(E)}\left[1-\exp \left[-\Sigma_{\mathrm{t}}(E) \delta\right]\right] \mathrm{d} E / E
$$

mit $\delta=$ Dicke der Goldfolie.

Unter Verwendung der mit dem Kristall-Spektrometer von Brookhaven für Gold erhaltenen Daten für $\sigma_{\mathrm{t}}$ und $\sigma_{\mathrm{s}} / \sigma_{\mathrm{t}}$ findet man als Korrektur für das Resonanzintegral $\int \sigma_{\mathrm{a}} \mathrm{d} E / E$ durch graphische Integration 52 barn, so daß das Resonanzintegral für Gold schließlich lautet

$$
R=1283 \pm 15 \text { barn } .
$$

${ }^{3}$ R. S. Carter, H. Palevsky, V. W. Myers u. D. J. Hughes, Phys. Rev. 92, 716 [1953].
Der angegebene Fehler trägt sowohl der Unsicherheit im thermischen Wirkungsquerschnitt von Gold als auch den Meßfehlern des Cd-Verhältnisses Rechnung.

Definiert man das Resonanzintegral als $\int_{0,5}^{\infty} \sigma_{\mathrm{eV}} \mathrm{d} E / E$, wobei $\sigma_{\mathrm{a}}$ der totale Absorptionsquerschnitt $\sigma_{\text {res }}+$ $\sigma_{1 / v}$ ist, so muß man zum oben angegebenen Wert noch

$$
\int_{0,5}^{\infty} \sigma_{0} \sqrt{E_{0} / E} \mathrm{~d} E / E=43 \text { barn }
$$

addieren, so daß das Resonanzintegral schließlich wird

$$
\begin{gathered}
R=\int_{0,5 \mathrm{eV}}^{\infty} \sigma_{\mathrm{a}} \mathrm{d} E / E=1326 \pm 15 \text { barn } . \\
\text { Schlußfolgerung }
\end{gathered}
$$

\section{Schlußfolgerung}

Das obige Resultat wurde ohne Verwendung der Wirkungsquerschnittskurve von Gold erhalten, abgesehen von der Korrektur für Selbstabsorption, die nur etwa $4 \%$ beträgt. Andererseits mußten die Daten für $\sigma_{\mathrm{t}}(E)$ von $\mathrm{Cd}$ oberhalb $0,3 \mathrm{eV}$ verwendet werden, was jedoch auf das endgültige Resultat nur einen kleinen Einfluß hat, da man sich erstens bereits ziemlich weit weg von der Cd-Resonanz befindet, und zweitens Unsicherheiten im Wirkungsquerschnitt nur schwach eingehen.

\title{
Zur Theorie des Kerr-Effekts in unpolaren Flüssigkeiten
}

\author{
Von Gerhard Klages*
}

Aus dem I. Physikalischen Institut der Johannes-Gutenberg-Universität Mainz

(Z. Naturforschg. 9 a, 602-607 [1954]; eingegangen am 10. Juni 1954)

\begin{abstract}
Ein dipolloses Molekül wird als Ellipsoid aus isotrop polarisierbarer Materie betrachtet und unter Zugrundelegung dieses Modells eine neue Beziehung für die Kerr-Konstante der Flüssigkeit abgeleitet. Als zusätzlicher Parameter erscheint darin die DK der Molekülmaterie, die größer als die makroskopische DK der Flüssigkeit ist und aus bekannten Werten für die Molekülvolumina abzuleiten ist. Es schließt sich eine Diskussion der schon vorliegenden Messungen des Kerr-Effekts von unpolaren Substanzen an, aus der die grundsätzliche Verwendbarkeit des Modells folgt. Größere Abweichungen bei nahezu kugelsymmetrischen Molekeln werden als zusätzlicher Schwankungseffekt in der Flüssigkeit gedeutet, während bei unpolaren Benzolderivaten noch eine anisotrop polarisierbare Molekülmaterie angenommen werden muß.
\end{abstract}

$\mathrm{I}_{\mathrm{n}}^{\mathrm{n}}$ einer früheren Arbeit $^{1}$ wurde gezeigt, daß bei Dipolsubstanzen die großen Differenzen zwischen den molaren Kerr-Konstanten, die man aus Messungen im Dampf und in der Flüssigkeit berechnet,

\footnotetext{
* Vorgetragen auf der Physiker-Tagung in Stuttgart am 2. 5. 1954 .

${ }^{1}$ G. Klages, Z. Naturforschg. 7a, 669 [1952].
}

in erster Linie vom nicht richtigen Ansatz des inneren Feldes herrühren. Überträgt man die Onsagersche Rechnung ${ }^{2}$ für das richtende Feld ${ }^{3}$, das auf einen Dipol innerhalb der Flüssigkeit einwirkt, auf

\footnotetext{
${ }^{2}$ L. Onsager, J. Amer. Chem. Soc. 58, 1486 [1936].

${ }^{3}$ s. C. J. F. Böttcher, Theory of Dielectric Polarisation, Elsevier Publ. Comp. 1952, S. 174.
} 
die Theorie der Kerr-Konstanten, so stimmen die beiden molaren Kerr-Konstanten wesentlich besser überein.

Auf unpolare Substanzen läßt sich nun aber die Onsagersche Überlegung nicht ohne weiteres übertragen, da man für $\varepsilon=n^{2}$ wieder das Lorentz-Feld

$$
E_{i}=\frac{n^{2}+2}{3} E_{0}
$$

erhält, mit dem die ältere Theorie des Kerr-Effekts auch rechnet. Das beruht im wesentlichen darauf, daß auch Onsager für unpolare Flüssigkeiten das mittlere Feld in einem Molekül gleich dem Mittelwert des lokalen Feldes über das ganze Dielektrikum setzt. - Außerdem erscheint es wenig sinnvoll, bei der Berechnung des Anisotropie-Gliedes, das ja allein die Kerr-Konstante unpolarer Molekeln bestimmt, die Form des Moleküls nicht zu berücksichtigen, indem man es mit Onsager als Kugel approximiert. Ähnlich wie Abbott und Bolton ${ }^{4}$ bei der Berechnung der dielektrischen Polarisation vorgehen, sollte daher auch hier das Molekül wenigstens als Ellipsoid angenähert werden.

\section{Theoretische Überlegungen}

Wir denken uns daher das Molekül als Ellipsoid, das aus einem homogenen Dielektrikum besteht, dessen Hauptbrechungsindizes $n_{\mathrm{M} i}$ in die Ellipsoidachsen $a_{i}$ fallen. Die $n_{\mathrm{M} i}$ sind also vom makroskopischen Brechungsindex in der Flüssigkeit verschieden, da in letzteren noch der Anteil der Zwischenräume zwischen den Molekeln eingeht. Die Hauptpolarisierbarkeiten des Moleküls im Vakuum sind dann

$$
\alpha_{i}=\frac{a_{1} a_{2} a_{3}}{3} \frac{n_{\mathbf{M} i}^{2}-1}{1+\left(n_{\mathbf{M} i}^{2}-1\right) A_{i}},
$$

wenn $A_{i}$ der Entelektrisierungsfaktor in Richtung der Achse $a_{i}$ ist.

\section{a) Induziertes Moment}

Befindet sich das Molekül nun innerhalb der Flüssigkeit, die als homogenes Medium mit dem Brechungsindex $n$ angenähert wird, und liegt die Feldstärke $E_{\mathrm{L}}$ einer Lichtwelle in Richtung der Achse $a_{i}$, so wird das induzierte Moment:

Dabei ist

$$
m_{i}=\frac{\alpha_{i}}{1-f_{i} \alpha_{i}} \frac{E_{\mathrm{L}}}{K_{i}}=\alpha_{i}^{*} E_{\mathrm{L}} .
$$

$$
\frac{E_{\mathrm{L}}}{K_{i}}=\frac{n^{2}}{n^{2}+\left(1-n^{2}\right) A_{i}} E_{\mathrm{L}}
$$

${ }^{4}$ J. A. Abbott u. H. C. Bolton, Trans. Faraday Soc. 48, 422 [1952]. das Hohlraumfeld in dem durch das Ellipsoidmolekül ausgefüllten Raum der Flüssigkeit, $f_{i}$ der Faktor der Rückwirkungsfeldstärke des induzierten Moments nach Scholte ${ }^{5}$

$$
f_{i}=\frac{3}{a_{1} a_{2} a_{3}} \frac{A_{i}\left(1-A_{i}\right)\left(n^{2}-1\right)}{n^{2}+\left(1-n^{2}\right) A_{i}} .
$$

Damit wird nach einigen Umrechnungen :

$$
\alpha_{i}^{*}=\alpha_{i} n^{2} \frac{1+\left(n_{\mathrm{M} i}^{2}-1\right) A_{i}}{n^{2}+\left(n_{\mathbf{M} i}^{2}-n^{2}\right) A_{i}} .
$$

Für $n_{\mathrm{M} i}^{2} \equiv n^{2}$ folgt daraus unter Heranziehung von (1), daß alle $\alpha_{i}{ }^{*}$ gleich sind, so daß für völlig homogene Materie die Anisotropie verloren geht, wie es auch sein muß.

\section{b) Potentielle Energie}

Das Ellipsoid liege nun mit seiner Achse $a_{i}$ in der Richtung eines Gleichfeldes $E_{0}$, wieder umgeben von einem kontinuierlichen Medium der makroskopischen DK $\varepsilon$. Die statischen Hauptpolarisierbarkeiten $\overline{\alpha_{i}}$ errechnen sich dann aus (1) unter Ersetzung von $n_{\mathbf{M} i}^{2}$ durch $\varepsilon_{\mathbf{M} i}$. Seine potentielle Energie im Felde $E_{0}$ ist allgemein die eines induzierten Dipols :

$$
U=-\frac{1}{2} m_{i}^{\mathrm{a}} E_{0}
$$

$m_{i}^{\mathrm{a}}$ ist dabei das sogenannte äußere Moment des polarisierten Ellipsoids in der Flüssigkeit, das sich nach der Potentialtheorie ergibt als:

$$
m_{i}^{\mathrm{a}}=\frac{a_{1} a_{2} a_{3}}{3} \frac{\varepsilon\left(\varepsilon_{\mathbf{M} i}-\varepsilon\right)}{\varepsilon+\left(\varepsilon_{\mathbf{M} i}-\varepsilon\right) A_{i}} E_{0} .
$$

Durch Einsetzung von $\overline{\alpha_{i}}$ aus (1) wird schließlich:

$$
\begin{gathered}
U=-\frac{1}{2} \overline{\alpha_{i}} \frac{\varepsilon_{\mathbf{M} i}-\varepsilon}{\varepsilon_{\mathbf{M} i}-1} \varepsilon \frac{1+\left(\varepsilon_{\mathbf{M} i}-1\right) A_{i}}{\varepsilon+\left(\varepsilon_{\mathbf{M} i}-\varepsilon\right) A_{i}} \cdot E_{0}{ }^{2} \\
=-\frac{1}{2} \overline{\alpha_{i}{ }^{*}} E_{0}{ }^{2} .
\end{gathered}
$$

c) Clausius-Mosottische Beziehung

Für die weitere Rechnung wollen wir unser Modell vereinfachen, indem wir die Molekülmaterie als isotrop polarisierbar annehmen, also alle $n_{\mathrm{M} i}=n_{\mathrm{M}}$ setzen. Wir denken uns damit die optische Anisotropie der Molekel allein durch ihre Form verursacht. Weiter sei bei den betrachteten unpolaren Stoffen nicht mehr zwischen optischer und statischer Polarisierbarkeit unterschieden und daher $\varepsilon=n^{2}$ angenommen.

Aus (2) und (3) kann man dann die Polarisation $P$ der Flüssigkeit berechnen und erhält daraus eine Beziehung für den Brechungsindex $n$ :

5 Th. G. Scholte, Physica 15, 437 [1949]. 


$$
\begin{aligned}
n^{2}-1=4 \pi \frac{P}{E_{\mathrm{L}}}= & 4 \pi N \frac{a_{1} a_{2} a_{3}}{3} \frac{n^{2}}{3} \\
& \cdot \sum_{i=1}^{3} \frac{n_{\mathrm{M}}^{2}-1}{n^{2}+\left(n_{\mathrm{M}}^{2}-n^{2}\right) A_{i}} .
\end{aligned}
$$

Bis auf einen Korrekturfaktor, der aber bei den später untersuchten Molekülen nur einige Prozent beträgt, kann man dann schreiben:

$$
\begin{array}{r}
n^{2}-1=4 \pi N \frac{a_{1} a_{2} a_{3}}{3}\left(n_{\mathrm{M}}^{2}-1\right) \frac{3 n^{2}}{2 n^{2}+n_{\mathrm{M}}^{2}} \\
=4 \pi N \alpha^{*}(n) .
\end{array}
$$

In dieser erweiterten Clausius-Mosottischen Gleichung wird also die Anisotropie der Moleküle vernachlässigt, da sie sich nur in Gliedern zweiter Ordnung in den $A_{i}$ bemerkbar macht. Die gefundene Additivität der Atompolarisationen liefert einen experimentellen Beweis für die Berechtigung dieser Vernachlässigung. In derselben Näherung wird die mittlere Polarisierbarkeit $\alpha$ des Moleküls im Vakuum:

$$
\alpha=\frac{1}{3} \sum_{i=1}^{3} \alpha_{i}=\alpha^{*}(1)=a_{1} a_{2} a_{3} \frac{n_{\mathrm{M}}^{2}-1}{n_{\mathrm{M}}^{2}+2} .
$$

d) Kerr-Konstante

Die Born-Langevinsche Rechnung ${ }^{6}$ für den KerrEffekt kann man nun direkt auf unsere Ausgangsgrößen $\alpha_{i}{ }^{*}$ und $\overline{\alpha_{i}{ }^{*}}$ übertragen. Man erhält dabei für die Brechungsindizes $n_{\mathrm{p}}$ und $n_{\mathrm{s}}$ parallel und senkrecht zum angelegten Gleichfeld $E_{0}$ :

$$
\begin{aligned}
& n_{\mathrm{p}}{ }^{2}-1=4 \pi N\left(\alpha^{*}\left(n_{\mathrm{p}}\right)+2 \Theta_{1}{ }^{*} \frac{E_{0}{ }^{2}}{2}\right), \\
& n_{\mathrm{s}}{ }^{2}-1=4 \pi N\left(\alpha^{*}\left(n_{\mathrm{s}}\right)-\Theta_{1}{ }^{*} \frac{E_{0}{ }^{2}}{2}\right)
\end{aligned}
$$

mit

$$
\begin{aligned}
\Theta_{1}{ }^{*} & =\frac{1}{45 k T}\left[\left(\alpha_{1}{ }^{*}-\alpha_{2}{ }^{*}\right)\left(\overline{\alpha_{1}{ }^{*}}-\overline{\alpha_{2}{ }^{*}}\right)\right. \\
& \left.+\left(\alpha_{2}{ }^{*}-\alpha_{3}{ }^{*}\right)\left(\overline{\alpha_{2}{ }^{*}}-\overline{\alpha_{3}{ }^{*}}\right)+\left(\alpha_{3}{ }^{*}-\alpha_{1}{ }^{*}\right)\left(\overline{\alpha_{3}{ }^{*}}-\overline{\alpha_{1}{ }^{*}}\right)\right] .
\end{aligned}
$$

Berücksichtigt man (5), so ergibt sich nach einigen Umrechnungen die Kerr-Konstante $K$ :

$$
K=\frac{n_{\mathrm{p}}-n_{\mathrm{s}}}{n} \frac{1}{{E_{0}{ }^{2}}^{2}}=3 \pi N \frac{2 n^{2}+n_{\mathrm{M}}^{2}}{2 n^{4}+n_{\mathrm{M}}^{2}} \Theta_{1}{ }^{*} \text {. }
$$

Den Ausdruck für $\Theta_{1}^{*}$ kann man durch Einsetzen der effektiven Polarisierbarkeiten aus (3) und (4) noch vereinfachen, was $\mathrm{zu}$ der endgültigen $\mathrm{Be}$ ziehung führt:

$$
K=\frac{\pi}{27} N X \Theta_{1}^{\prime},
$$

${ }^{6}$ Literaturzitate s. 1. c. ${ }^{1}$.

7 Meßwerte aus H. A. Stuart, Hand- u. Jahrbuch wobei der Feldfaktor $X$ sich zu

$$
\begin{aligned}
& X=\frac{81 n^{4}}{\left(Y_{1} Y_{2} Y_{3}\right)^{2}} \frac{n_{\mathrm{M}}^{2}+2}{n_{\mathrm{M}}^{2}+2 n^{4}}\left[\frac{\left(n_{\mathrm{M}}^{2}-n^{2}\right)\left(n_{\mathrm{M}}^{2}+2\right)}{\left(n_{\mathrm{M}}^{2}-1\right)\left(n_{\mathrm{M}}^{2}+2 n^{2}\right)}\right]^{3}, \\
& Y_{i}=1+\frac{\alpha_{i}-\alpha}{\alpha} \frac{n^{2}-1}{n_{\mathrm{M}}^{2}-1} \frac{3 n_{\mathrm{M}}^{2}}{2 n^{2}+n_{\mathrm{M}}^{2}}
\end{aligned}
$$

ergibt, während nach der alten Theorie, die das Lorentz-Feld verwendet,

$$
X_{\mathrm{L}}=\frac{\left(n^{2}+2\right)^{4}}{n^{2}}
$$

betrug. Weiter ist:

$$
\begin{aligned}
\Theta_{1}^{\prime}=\frac{1}{45 k T}\left[\left(\alpha_{1}-\alpha_{2}\right)^{2} Y_{3}{ }^{2}\right. & +\left(\alpha_{2}-\alpha_{3}\right)^{2} Y_{1}{ }^{2} \\
& \left.+\left(\alpha_{3}-\alpha_{1}\right)^{2} Y_{2}{ }^{2}\right] .
\end{aligned}
$$

Für den Gaszustand wird mit $n^{2}=1$ natürlich übereinstimmend $X=X_{\mathrm{L}}=81$. Mit wachsendem $n$, also dichterer Packung der Moleküle, wird jedoch $X_{\mathrm{L}}$ in steigendem Maße größer als $X$ aus (8). Berechnet man also die molare Kerr-Konstante $M K=$ $\pi / 27 \cdot N_{\mathrm{L}} \Theta_{1}$ aus den Meßwerten der Flüssigkeit, so müssen bei Verwendung von Beziehung (8) größere Werte herauskommen, so daß die früheren Diskrepanzen mit in der Flüssigkeit verkleinerter $M K$ auch bei unpolaren Substanzen zunächst einmal auf dem bei der Auswertung verwendeten, nicht richtigen Feldfaktor $X_{\mathrm{L}}$ beruhen dürften.

\section{Anwendung auf das vorliegende Beobachtungsmaterial}

a) Reine unpolare Flüssigkeiten

In den neuen Beziehungen für die Kerr-Konstante erscheint als zunächst unbekannte Größe $n_{\mathrm{M}}^{2}$, und wir wollen bei der Auswertung des experimentellen Materials so vorgehen, daß wir zunächst einmal $n_{\mathrm{M}}^{2}$ so bestimmen, daß die $M K$ für Gas und Flüssigkeit gleich werden. In Tab. 1 sind dazu die Meßwerte ${ }^{7} K$ der Flüssigkeit sowie die daraus nach der alten Theorie berechneten molaren Kerr-Konstanten $M K_{\mathrm{L}}$, in den nächsten beiden Spalten $M K$ und optische Anisotropie $\delta^{2}$ aus Gasmessungen angegeben. Die Größe $M K_{\mathrm{L}} / M K$ stellt den formalen ,Reduktionsfaktor" der Kerr-Konstanten in der Flüssigkeit dar, wenn man das Lorentz-Feld zur Auswertung heranzieht. Die Berechnung von $n_{\mathrm{M}}^{2}$, das in der letzten Spalte angegeben ist, erweist sich als ziemlich kritisch, indem 5\% Meßfehler in einer der beiden zugrunde gelegten gemessenen KerrKonstanten eine Änderung von etwa 0,1 ergibt.

der chem. Physik Bd. 10/III, Leipzig 1939, S. 47 ff., $64,103$. 
In diesem Sinne darf man $n_{\mathbf{M}}^{2}$ für die normalen Kohlenwasserstoffe als konstant ansehen, wie man es auch nach ihrem gleichen Auf bau erwarten sollte. Der Gang der ,,Reduktionsfaktors “ mit der Kettenlänge liegt wohl im steigenden $n^{2}$ begründet, was $\mathrm{zu}$ einer Verkleinerung der mit $X_{\mathrm{L}}$ berechneten $M K_{\mathrm{L}}$ führt. Es besteht keine Notwendigkeit, auf eine wachsende Parallelisierung und Verknäuelung der Kettenmoleküle in der Flüssigkeit zu schließen, wie früher angenommen wurde ${ }^{8}$. halten. Es wird dabei nämlich statt auf $n^{2}=1$ auf das $n^{2}$ des Lösungsmittels extrapoliert, wodurch man infolge des für $n^{2}>1$ immer zu großen Feldfaktors $X_{\mathrm{L}}$ der alten Theorie immer eine zu kleine molare Kerr-Konstante berechnet.

\section{Diskussion}

Die entwickelte Theorie der Kerr-Konstanten hat nun den Vorteil, daß die neu eingeführte Größe $n_{\mathrm{M}}^{2}$ nicht nur aus den Meßwerten des Kerr-Effekts in

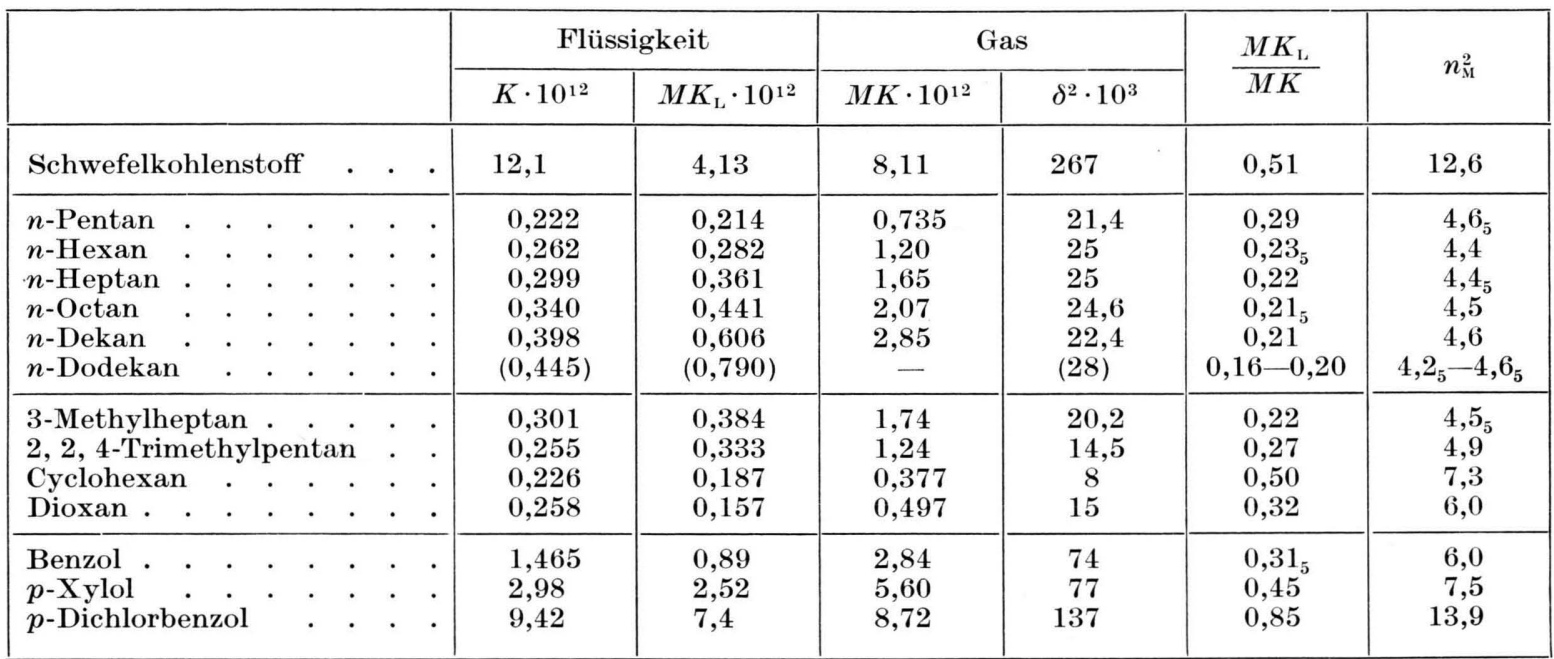

Tab. 1. Kerr-Konstanten in der Flüssigkeit und im Gas. Berechnung der DK der Molekülmaterie $n_{\mathrm{M}}^{2}$. Temperatur $20^{\circ} \mathrm{C}$. Erklärung der Bezeichnungen im`Text.

b) Mischungen unpolarer Flüssigkeiten

Ziehen wir nun die Messungen von Briegleb ${ }^{9}$ an Benzol in Mischungen mit $n$-Heptan zur weiteren Prüfung der Theorie heran, so können wir für $n_{\mathrm{M}}^{2}$ die Werte der reinen Flüssigkeiten aus Tab. 1 verwenden. Wie Tab. 2 zeigt, wird dann die $M K$ von Benzol, aufgeführt in der letzten Spalte, unabhängig von der Konzentration. Auch hier kann man also nicht auf eine spezielle Wechselwirkung oder Ordnung der Benzol-Molekeln schließen, die mit steigender Verdünnung aufgelöst wird, wie früher der Gang von $M K_{\mathrm{L}}$ gedeutet wurde. Letzterer rührt vielmehr allein im Sinne der obigen Diskussion von dem mit der Verdünnung absinkenden $n^{2}$ der Mischung her. Man sieht daran, daß es keinen Sinn hat, $M K_{\mathrm{L}}$ auf unendliche Verdünnung zu extrapolieren, um die richtige, d. h. mit dem Gaswert vergleichbare molare Kerr-Konstante zu er-

${ }^{8}$ H. A. Stuart, 1. c. ${ }^{7}$, S. 102. der im vorigen Abschnitt erläuterten Art gewonnen und ihr Verhalten in homologen Reihen und bei Mischungen diskutiert werden kann. Bei Anwen-

\begin{tabular}{|c|c|c|c|}
\hline$c$ & $n^{2}$ & $M K_{\mathrm{L}} \cdot 10^{12}$ & $M K \cdot 10^{12}$ \\
\hline 0,150 & 1,99 & 1,25 & 2,86 \\
0,268 & 2,01 & 1,19 & 2,87 \\
0,471 & $2,05_{5}$ & 1,14 & 2,89 \\
0,603 & 2,10 & 1,08 & 2,89 \\
0,725 & 2,15 & 1,04 & 2,95 \\
0,865 & 2,20 & 0,95 & 2,86 \\
1,000 & $2,25_{5}$ & $0,89_{5}$ & 2,84 \\
\hline
\end{tabular}

Tab. 2. Mischungen von Benzol mit $n$-Heptan nach G. B r i e g l e b. c Molenbruch von Benzol.

dung der Beziehung (5) ist sie vielmehr auch auf anderem Wege zu berechnen, wenn das Molekülvolumen bekannt ist. In Tab. 3 sind dazu herangezogen der gaskinetische Durchmesser der Moleküle

${ }^{9}$ G. Briegleb, Z. phys. Chem. (B) 14, 97 [1931]. 
aus Viskositäts-Messungen bei Extrapolation auf $1 / T=0$ sowie das nach van der Waals berechnete Eigenvolumen der Moleküle aus den kritischen Daten ${ }^{10}$. Leider ist dieses Volumen für die Berechnung von $n_{\mathrm{M}}^{2}$ sehr kritisch, und die einzelnen Werte streuen sehr stark, schon bei den Volumina selbst, was man besonders an den normalen Kohlenwasserstoffen sehen kann. Daher ist für sie noch der Mittelwert aus allen gemessenen Gliedern der homologen Reihe angegeben. Die ebenfalls aufgeführten Werte,

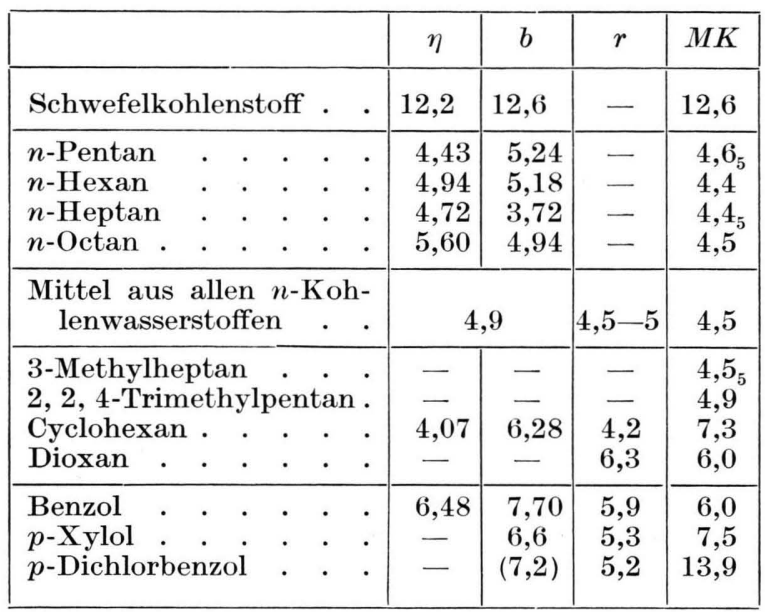

Tab. 3. DK der Molekülmaterie berechnet mit Hilfe der Eigenvolumina der Moleküle aus: $\eta$ Viskosität, $b$ kritischen Daten, $r$ Bindungsradien sowie mit Hilfe der molaren Kerr-Konstanten $(M K)$.

die auf einer Abschätzung des Molekülvolumens aus den Bindungsradien mit 0,5 AE Wirkungsradius an der Oberfläche beruhen, sind noch unsicherer und können nur bei Molekülen mit sonst unbekanntem Volumen als Richtwerte angesehen werden.

Beim Vergleich der so erhaltenen DK der Molekülmaterie mit der aus dem Kerr-Effekt berechneten aus Tab. 1 ergibt sich gute Übereinstimmung bei Schwefelkohlenstoff und den normalen Kohlenwasserstoffen, so daß für diese Moleküle das vereinfachte Modell der isotrop polarisierbaren Molekülmaterie anwendbar ist. Sehr große Abweichungen dagegen zeigt Cyclohexan, bei dem die Kerr-Konstante der Flüssigkeit nach dem $n_{\mathrm{M}}^{2}$ der ersten drei Spalten von Tab. $3 \mathrm{zu}$ groß ist. Gleichzeitig besitzt dieses Molekül die kleinste optische Anisotropie, nähert sich also sehr stark der Kugelsymmetrie.

${ }^{10}$ Landolt-Börnstein, Atom- u. Molekularphysik, Teil 1. S. $369 \mathrm{ff}$.
Nun zeigt Tetrachlorkohlenstoff in der Flüssigkeit auch einen gut meßbaren Kerr-Effekt, obwohl das Molekül nach Gasmessungen isotrop polarisierbar ist. Dieser Kerr-Effekt kann daher nicht von einer molekularen Anisotropie herrühren, sondern nur von statistischen Schwankungen in der Flüssigkeit, bei denen nach Peterlin und Stuart ${ }^{11}$ zufällig anisotrop gewordene Volumenelemente im Felde orientiert werden. Einen solchen Zusatzeffekt durch Schwankungserscheinungen könnte man daher auch beim Cyclohexan erwarten und ihn in Analogie zu den Beobachtungen von $W$ hiffen ${ }^{12}$ bei der anomalen Absorption von unpolaren Flüssigkeiten im cmWellenbereich als Bevorzugung der Schwankungen ansehen, die im angelegten Feld energetisch günstig sind, also zu einer positiven Anisotropie der Volumenelemente führen. Beim nicht ganz so kugelsymmetrischen Trimethylpentan deutet sich dieser Zusatzeffekt schon an, während Methylheptan ihn nicht mehr zeigt, so daß zu vermuten ist, daß er mit zunehmender Anisotropie der Molekeln sehr schnell abnimmt.

Für Dioxan und Benzol dürfte in erster Näherung das vereinfachte Modell genügen, wohingegen bei den beiden $p$-Verbindungen des Benzols wieder beträchtliche Abweichungen auftreten. Beide haben ungefähr gleiches Volumen und gleiche Form, während die Hauptpolarisierbarkeiten der Moleküle, wie sie Stuart ${ }^{7}$ aus Kerr-Effekt und Depolarisationsgrad des Streulichts im Dampf bestimmt hat (Tab. 4), sehr verschieden sind. Letztere lassen sich also nicht mehr allein aus der Molekülform ableiten,

\begin{tabular}{|l|l|l|l|l|}
\hline & $\alpha \cdot 10^{24}$ & $\alpha_{1} \cdot 10^{24}$ & $\alpha_{2} \cdot 10^{24}$ & $\alpha_{3} \cdot 10^{24}$ \\
\hline Benzol & 103,2 & 123,1 & 123,1 & 63,5 \\
$\begin{array}{l}p \text {-Xylol } \\
p \text {-Dichlorbenzol }\end{array}$ & 142 & 182 & 156 & 88 \\
& 144,7 & 212,9 & 124,8 & 88,3 \\
\hline
\end{tabular}

Tab. 4. Hauptpolarisierbarkeiten der Benzolderivate nach H. A. Stuart.

was auch eine Abschätzung der Entelektrisierungsfaktoren aus der Molekülform zeigt. Vielmehr ist die Molekülmaterie beim $p$-Dichlorbenzol in Richtung der Substituenten, beim $p$-Xylol senkrecht dazu in der Ringebene besonders gut polarisierbar mit entsprechend höheren $n_{\mathrm{M} i}^{2}$ für diese Richtungen. Betrachtet man $\alpha_{i}^{*}$ und $\overline{\alpha_{i}^{*}}$ nach (3) und (4),

11 A. Peterlin u. H. A. Stuart, Z. Phys. 113, 663 [1939].

${ }^{12}$ D.H.Whiffen, Trans. Faraday Soc.46, 124 [1950]. 
so sieht man den großen Einfluß von $n_{\mathbf{M} i}^{2}$ auf diese Größen, der bei beiden Molekeln eine verschiedene Vergrößerung von $\Theta_{1}{ }^{*}$ verursachen dürfte, gegenüber den Werten, die man mit einem einheitlichen $n_{\mathrm{M}}^{2}$ erhält, wie es unserem vereinfachten Modell entspricht.

Abgesehen von den Schwankungserscheinungen kann also eine elektrostatische Theorie den KerrEffekt auch in unpolaren Flüssigkeiten wenigstens in ihren Grundzügen richtig darstellen. Die durchgeführten Überlegungen zeigen, daß der Grund für die Diskrepanzen zwischen Flüssigkeits- und Gaswerten nach der alten Theorie vornehmlich in der Ersetzung des inneren und des richtenden Feldes durch den Feldmittelwert über die ganze Substanz (Lorentz-Feld) zu suchen ist. Beim Vergleich mit dem Beobachtungsmaterial ergibt sich keine Notwendigkeit, den Einfluß einer besonderen Flüssigkeitsstruktur, die mit wachsender molekularer Anisotropie die Kerr-Konstante herabsetzen sollte, als für den Kerr-Effekt wesentlich bestimmend anzunehmen. Vielleicht hat man sich doch bisher über das Ausmaß der Parallellagerung von unpolaren Molekülen in der Flüssigkeit bei niedermolekularen Substanzen eine etwas übertriebene Vorstellung gemacht, zumal wenn man bedenkt, daß nach den Volumenabschätzungen das Eigenvolumen der Moleküle nur 0,3 bis 0,4 des ganzen Flüssigkeitsvolumens ausmacht und die Molekeln im Raum um drei Achsen drehbar sind.

Man kann zwar auch jetzt nicht ohne weiteres die molekulare optische Anisotropie aus KerrEffektsmessungen an Flüssigkeiten ableiten, da dazu die Größen $n_{\mathrm{M} i}^{2}$ bekannt sein müßten. Der Vergleich von Flüssigkeits- und Gasmessungen dürfte aber auf eine andere und bessere Grundlage gestellt sein, indem man dabei gerade Aussagen über die $n_{\mathbf{M} i}^{2}$ und damit solche zur Frage der anisotropen Polarisierbarkeit besonders von substituierten Gruppen gewinnen kann.

\title{
Eine photoelektrische Methode zur gleichzeitigen Bestimmung von Lebensdauer und Beweglichkeit injizierter Stromträger in Halbleitern
}

\author{
Von Günter AdaM
}

\begin{abstract}
Aus dem Institut für theoretische und angewandte Physik der Technischen Hochschule Stuttgart
\end{abstract}

(Z. Naturforschg. 9a, 607-611 [1954]; eingegangen am 9. April 1954)

\begin{abstract}
Mit Hilfe eines schwenkbaren Lichtbündels wird in Germanium ein gleichförmig fortbewegtes Generationsgebiet für zusätzliche Stromträger geschaffen. Die unsymmetrische stationäre Trägerverteilung, die sich um die Einstrahlungsstelle herum ausbildet, liefert an einer Spitzensonde eine proportionale Photospannung, die oszillographisch registriert wird. Die Verteilung wird berechnet, und es werden Verfahren zur Auswertung der erhaltenen Oszillogramme abgeleitet und an Beispielen erläutert. Lebensdauer und Diffusionskonstante lassen sich aus einem Oszillogramm ermitteln. Meßergebnisse an verschieden dotierten einkristallinen Germaniumproben werden mitgeteilt.
\end{abstract}

$\mathrm{W}$ ird in Germanium Licht eingestrahlt, so werden durch Anregung von Elektronen aus dem Valenzband ins Leitfähigkeitsband paarweise Leitungselektronen und Löcher erzeugt. Diese überschüssigen Stromträgerpaare diffundieren aus dem Gebiet der Lichtabsorption, wo sie erzeugt werden, in die unbeleuchtete Umgebung und verschwinden nach einer mittleren Lebensdauer $\tau$ durch Rekombination.

${ }^{1}$ F. S. Goucher, Phys. Rev. 81, 475 [1951].

2 Nach Bardeen gilt die Proportionalität exakt für den Photokurzschlußstrom, für die Photospannung
Der sich ausbildende Dichteverlauf kann nach Goucher ${ }^{1}$ durch Aufsetzen einer Spitze auf die Germanium-Oberfläche festgestellt werden. An der dem Spitzenkontakt vorgelagerten Sperrschicht entsteht eine Photospannung, die der überschüssigen Paardichte in der unmittelbaren Umgebung der Spitze proportional ist ${ }^{2}$. Verwendet man tief eindringendes Licht und schaltet man Oberflächenrekombination durch Ätzen der Oberfläche weit-

aber nur näherungsweise bei schwacher Injektion. Vgl. J. Bardeen, Bell. Syst. Techn. J. 31469 [1950], Gln. (7) und (12). 